\title{
RECOVERING THE INTRINSIC METALLICITY DISTRIBUTION OF ELLIPTICAL GALAXIES
}

\author{
L. CIOTTI \\ Osservatorio Astronomico di Bologna \\ via Zamboni 33, I-40126 Bologna \\ M. STIAVELLI \\ Scuola Normale Superiore \\ P.zza de' Cavalieri 7, I-56126 Pisa
}

AND

\section{A. BRACCESI}

Dipartimento di Astronomia, Università di Bologna via Zamboni 33, I-40126 Bologna

We present a simple recipe to derive the metallicity distribution of galaxies as a function of their integrals of the motion. Elliptical galaxies are known to possess metallicity gradients that frequently show variations of a factor of 2 from the center to the effective radius (Peletier, 1989). The observed gradients are the result of projection and orbital mixing. Orbital mixing arises because stars at a given radius may have their apocenters spanning a wide range of radii. Thus, the mean metallicity at any given point inside the galaxy is the result of the metallicity distribution in phase space weighted by the galaxian distribution function. We have proposed (Ciotti, Stiavelli \& Braccesi, 1995) a simple inversion procedure allowing one to derive the dependence of metallicity on the integrals of motion for a spherical galaxy. The ideas of this paper can be generalized to oblate two-integrals models following the Hunter \& Qian (1993) technique.

\section{References}

Ciotti, L., Stiavelli, M., and Braccesi, A., (1995), in press on M.N.R.A.S.

Hunter, C., Qian, E., (1993), M.N.R.A.S., 262, 401

Peletier, R., (1989), PhD Thesis (Groningen) 